\title{
REDES VERTICALES VERSUS HORIZONTALES Y DESEMPEÑO DE LAS EXPORTACIONES EN LA INDUSTRIA VITIVINÍCOLA ESPAÑOLA
}

\author{
$\underline{\left.\text { Juan-Ramón Ferrer }{ }^{\mathrm{a}}{ }^{*}\right)}$, Silvia Abella-Garcés ${ }^{\mathrm{b}}$, Raúl Serrano ${ }^{\mathrm{b}}$
}

a Universidad Politécnica de Madrid (Madrid, juanramon.ferrer@upm.es). ${ }^{b}$ Universidad de Zaragoza
(Zaragoza, sabella@unizar.es,raser@unizar.es)

\section{Resumen}

Las bodegas españolas apuestan por la internacionalización, ya que los mercados locales no tienen capacidad para absorber su producción. Pero solo las empresas más grandes con una mayor productividad pueden cubrir los costos fijos necesarios para ingresar a los mercados de exportación. La teoría de redes sugiere que las empresas pueden superar sus limitaciones de recursos y capacidad cooperando con otras empresas. La pertenencia a una red puede permitir a las empresas adquirir el conocimiento que necesitan sobre los mercados internacionales potenciales, obtener los recursos necesarios para penetrar en ellos y lograr sinergias a través de costos y riesgos.

Este estudio busca determinar qué tipo de red (vertical u horizontal) tiene un mayor impacto en el éxito internacional de la firma. Nos basamos en una muestra de 182 bodegas españolas y examinamos las principales variables independientes mediante un modelo de Heckman. Encontramos que la participación en redes tiene un efecto positivo en la intensidad de las exportaciones, y resultando especialmente relevante las redes horizontales, mientras que en las verticales destacan las descendentes sobre las ascendentes.

Palabras clave: Desempeño exportador, Redes, sector vitivinícola español.

\section{Introducción}

De acuerdo con el proceso de internacionalización de Uppsala (Vahlne y Johanson, 2017), la mejor manera de que las pymes ingresen a los mercados internacionales es a través de las redes nacionales, ya que el menor tamaño de las pymes es un obstáculo para ingresar a las redes internacionales. Dentro de las redes diferenciamos entre redes verticales y horizontales. Los primeros se forman entre empresas a lo largo de la cadena de suministro e incluyen proveedores y clientes. Algunos estudios muestran que las redes verticales mejoran el desempeño de las exportaciones más que las horizontales y, por lo tanto, las empresas deben enfocarse en la red de la cadena para establecer las mejores relaciones y la confidencialidad dentro de su propia red de la cadena (Easmon et al., 2019). Las redes horizontales se refieren a las relaciones entre empresas que compiten en un mismo mercado que comparten sus capacidades con sus competidores para desarrollar conjuntamente nuevos productos e innovaciones, operando mejor con la formación de consorcios de producción, como la Denominación de Origen Europea (DOs) (Gellynck y Kuhne, 2010); cuanto más frecuentes y fuertes son los lazos, más eficientes son las redes y más innovadoras las empresas que participan en ellas (Franken et al., 2018).

\section{Objetivo}

El objetivo de este estudio es determinar en el sector vitivinícola español, qué tipo de redes, horizontales o verticales son más relevantes para favorecer la internacionalización de la empresa. Utilizamos el modelo de Heckman en dos etapas. La primera etapa estudia la influencia de los modelos de colaboración en la decisión de las empresas de exportar o no exportar. La segunda etapa analiza el efecto de los mecanismos de cooperación, las redes, sobre la intensidad exportadora.

\section{Metodología}

El universo de empresas utilizadas en el estudio está formado por bodegas españolas derivadas de la combinación de dos bases de datos diferentes. El primero contiene información sobre las DOP e IGP, recopilada por el Ministerio de Agricultura, Pesca y Alimentación (MAGRAMA); el segundo es el Sistema de Análisis de Balances Ibéricos (SABI), del que hemos tomado las empresas pertenecientes al apartado 11.02 de la Clasificación de Actividades Económicas (CNAE). El tamaño de la población es de 2.413 bodegas. Con el fin de obtener la información necesaria para el análisis, se envió un cuestionario a los gerentes de estas firmas solicitando información sobre la situación de las firmas en diciembre de 2015. El proceso se desarrolló entre febrero y mayo de 2016. Hubo un total de 264 cuestionarios (de los cuales 182 respondieron preguntas relacionadas con las exportaciones).

\subsection{Variables}

Variables dependientes. Se analizan dos variables dependientes: propensión a exportar e intensidad exportadora. La primera variable, propensión a exportar, tiene en cuenta si una empresa de la muestra 
exporta o no. Se mide mediante una variable ficticia que toma el valor 1 si la empresa i exportó en el año t, y cero en caso contrario. La segunda variable, la participación de las exportaciones, indica, una vez que una empresa exporta, cuánto exporta. Esto se calcula como el logaritmo de la relación entre las ventas de exportación y las ventas totales (LExport).

Variables independientes. Como variables independientes incluimos cuatro tipos de redes, dos verticales y dos horizontales. La variable de red horizontal (Red Horizontal 1), se mide a través de dos preguntas: 1) Participación en proyectos de $\mathrm{I}+\mathrm{D} ; 2$ ) Colaboración con organismos públicos de investigación u otras empresas. Los gerentes de las bodegas evalúan la posición de su empresa con respecto a sus competidores en una escala Likert de 5 puntos donde 1 es "mucho más débil que los competidores" y 5 es "mucho más fuerte que los competidores".

Una segunda variable utilizada para medir la participación en redes horizontales es la Red Horizontal 2, que indica si una empresa ha colaborado con otros competidores participando en una Denominación de Origen. Esta variable se mide por el número de DO en las que participa la bodega.

La tercera variable de red corresponde a red vertical upstream (Red Vertical 1), que es una medida de relaciones y colaboraciones arriba de la cadena de valor y se calcula a través de la pregunta: "establecer acuerdos y alianzas con proveedores". Los gerentes de las bodegas evalúan la posición de su empresa con respecto a sus competidores en una escala Likert de 5 puntos donde 1 es "mucho más débil que los competidores" y 5 es "mucho más fuerte que los competidores". Finalmente, para medir las relaciones y la colaboración downstream calculamos la variable de la red hacia abjo de la cadena de valor (Red Vertical 2), la cual se mide a través de dos preguntas: 1) "establecer acuerdos y alianzas con distribuidores convencionales", 2) "establecer acuerdos y alianzas con grandes- distribuidores ". Los gerentes de las bodegas evalúan la posición de su empresa con respecto a sus competidores en una escala Likert de 5 puntos donde 1 es "mucho más débil que los competidores" y 5 es "mucho más fuerte que los competidores".

Variables de control de la empresa. Se analizan, el tamaño de la empresa, la edad y la disponibilidad de recursos (tecnológicos, de innovación, recursos humanos, capacidades directivas, marketing y financieros). Todos los recursos se evalúan mediante la técnica de componentes principales, sobre los valores de las variables que se han generado mediante la opinión de los gerentes de las compañías sobre la posición de su empresa. Para cada una de estas variables, los gerentes de las bodegas evalúan la posición de su empresa con respecto a sus competidores en una escala Likert de 5 puntos donde 1 es "mucho más débil que los competidores" y 5 es "mucho más fuerte que los competidores".

\subsection{Modelo de Heckman-Probit}

La primera etapa del modelo incluye a todas las empresas que respondieron el cuestionario, 183 empresas. (incluidos 160 exportadores y 23 no exportadores), la segunda etapa solo incluye a las empresas exportadoras (160). En nuestro caso, la muestra está acorde con la realidad del sector en términos de tamaño de negocio, así como en términos de representatividad, ya que representa alrededor del 10\% del sector.La primera ecuación de Heckman se estima mediante un modelo de probabilidad (Probit) que analiza los factores que afectan la probabilidad de exportar.

$\mathrm{D}^{\exp _{\mathrm{i}, \mathrm{t}}}=\left\{\begin{array}{l}1 \text { (export }) \Longrightarrow \mathrm{P}\left(\mathrm{D}_{\mathrm{i}, \mathrm{t}}=1\right)=\mathrm{f}(\text { Estrategias }+ \text { variables de control }) \\ 0 \text { (no export) en otro caso }\end{array}\right.$

Donde la variable dependiente $\left(D_{i, t}^{e x p}\right)$ es una variable dummy que toma el valor 1 si la empresa $\mathrm{i}$ exporta en el año t, y cero si no lo hace.

En la segunda etapa del modelo, ecuación (2), se analiza los determinantes de la intensidad de exportación:

$$
\begin{aligned}
& \text { 1_Export,t }=\beta_{1} \text { Tipo de red }_{i}+\beta_{2} \text { Tamaño }_{i}+\beta_{3} \text { Edad }_{i}+\beta_{4} \text { Tecnologia }_{i}+\beta_{5} \text { Innovacion }_{i}+ \\
& +\beta_{6} \text { RRHH }_{i}+\beta_{7} \text { Gestión }_{i}+\beta_{8} \text { Market }_{i}+\beta_{9} \text { Finan }_{i}+\beta_{10} \text { Mills ratio }_{i}+U_{i j}
\end{aligned}
$$

\section{Resultados}

Los resultados de las estimaciones del modelo se muestran en el Cuadro 1. Se han realizado 4 modelos, que corresponden a los 4 tipos de redes estudiadas. Red vertical 1 upstream (columnas 1 y 2), red vertical 2 
downstream (columnas 3 y 4), red horizontal 1, colaboración investigación e instituciones (columnas 5 y 6), y red horizontal 2, colaboración con competidores (columnas 7 y 8). Para cada uno de los cuatro tipos de redes, presentamos dos modelos, ocho resultados en total. Uno para la primera etapa de la ecuación de selección de Heckman y otro para la ecuación de regresión de la segunda etapa. Los resultados apoyan el efecto positivo de la participación en determinadas redes y el desempeño exportador.

Cuadro 1. Efectos de las redes en la exportación y su intensidad

\begin{tabular}{|c|c|c|c|c|c|c|c|c|}
\hline & \multicolumn{2}{|c|}{ Red vertical 1} & \multicolumn{2}{|c|}{ Red vertical 2} & \multicolumn{2}{|c|}{ Red horizontal 1} & \multicolumn{2}{|c|}{ Red horizontal 2} \\
\hline & $\begin{array}{c}(1) \\
\text { Etapa } 1\end{array}$ & $\begin{array}{c}(2) \\
\text { Etapa } 2\end{array}$ & $\begin{array}{c}(3) \\
\text { Etapa } 1\end{array}$ & $\begin{array}{c}(4) \\
\text { Etapa } 2\end{array}$ & $\begin{array}{c}(5) \\
\text { Etapa } 1\end{array}$ & $\begin{array}{c}(6) \\
\text { Etapa } 2\end{array}$ & $\begin{array}{c}(7) \\
\text { Etapa } 1\end{array}$ & $\begin{array}{c}(8) \\
\text { Etapa } 2\end{array}$ \\
\hline Tipo de red & 0.170 & 0.222 & -0.041 & $0.169^{*}$ & -0.108 & -0.084 & 0.466 & $0.255^{* *}$ \\
\hline Tamaño & $0.308 * *$ & $0.476^{* * *}$ & $0.316^{* *}$ & $0.452 * * *$ & $0.386^{* * *}$ & $0.607 * * *$ & $0.354 * * *$ & $0.561 * * *$ \\
\hline Edad & $-0.376^{* *}$ & $0.643 * * *$ & $-0.340 * *$ & $0.633 * * *$ & $-0.264 * *$ & $0.540^{* * *}$ & $-0.303 * *$ & $0.556 * * *$ \\
\hline Tecnología & 0.012 & -0.052 & 0.044 & -0.024 & 0.040 & -0.005 & 0.066 & 0.038 \\
\hline Innovación & 0.101 & 0.207 & 0.148 & 0.170 & 0.177 & $0.436 * *$ & 0.141 & 0.272 \\
\hline $\boldsymbol{R R H H}$ & -0.069 & & 0.016 & & -0.125 & & -0.145 & \\
\hline Gestión & 0.153 & 0.255 & 0.070 & 0.247 & 0.142 & $0.289^{*}$ & 0.121 & $0.296^{*}$ \\
\hline Marketing & 0.176 & -0.238 & 0.253 & -0.301 & $0.301 *$ & -0.033 & 0.242 & -0.088 \\
\hline Finanzas & $-0.412 * *$ & -0.054 & $-0.390 * *$ & -0.025 & $-0.383 * *$ & -0.190 & $-0.321 * *$ & -0.173 \\
\hline Constante & $2.928 * * *$ & $13.678 * * *$ & $3.374 * * *$ & $13.405 * * *$ & $3.261 * * *$ & $14.772 * * *$ & $2.149 * *$ & $14.040 * * *$ \\
\hline Observaciones & 181 & 153 & 179 & 151 & 181 & 154 & 182 & 154 \\
\hline \multirow{2}{*}{ Mills lambda } & & -0.419 & & -0.227 & & 0.706 & & 0.731 \\
\hline & & $\mathrm{P}>|0,74|$ & & $\mathrm{P}>|0,86|$ & & $\mathrm{P}>|0,59|$ & & $\mathrm{P}>|0,55|$ \\
\hline Wald chi2 & & 66.42 & & 67.99 & & 85.87 & & 94.97 \\
\hline Prob > chi2 & & 0 & & 0 & & 0 & & 0 \\
\hline
\end{tabular}

Note: The level of statistical significance, $* * *, * *$ and $*$, denote 1,5 and 10 percent respectively.

En concreto, la participación en redes horizontales configuradas por DO (Red Horizontal 2), y las redes verticales, colaboración con la distribución comercial hacia abajo en la cadena de valor (Red Vertical 2), que incluye colaboraciones con distribuidores, tienen un efecto positivo en la intensidad exportadora. Respecto a la primera etapa, en las ecuaciones de selección no encontramos, para ningún tipo de red, un efecto estadísticamente significativo (columnas 1, 3, 5 y 7). Destacar el éxito de las redes de colaboración horizontal con la competencia, medidas como pertenecientes a las DO. También vale la pena señalar la importancia, aunque menor, de las redes de marketing vertical descendentes. Otra consideración necesaria se refiere a la primera fase del modelo, en la que no aparecen redes relevantes. Sin embargo, hay que destacarlo ya que prácticamente todas las empresas vitivinícolas de España exportan (más del 70\%), por lo que se trata de una decisión general que no está condicionada por el tipo de redes a las que pertenezcan estas firmas.

\section{Conclusiones}

El estudio pone de manifiesto como la pertenencia de las bodegas a diferentes redes de colaboración tiene un efecto positivo en su volumen de exportación, destacando las redes horizontales de colaboración dentro 
de la DOP y las redes verticales downstream de marketing.

\section{Bibliografía}

Easmon, R. B., Kastner, A. N. A., Blankson, C. y Mahmoud, M. A. (2019). "Social capital and export performance of SMEs in Ghana: the role of firm capabilities". African Journal of Economic and Management Studies, 10(3), 262-285.

Franken, J., Gómez, M., y Ross, R. B. (2018). "Social Capital and Entrepreneurship in Emerging Wine Regions". Journal of Wine Economics, 13(4), 419-428.

Gellynck, X. y Kühne, B. (2010). "Horizontal and vertical networks for innovation in the traditional food sector". International Journal on Food System Dynamics, 1(2), 123-132.

Lacam, J. S. y Salvetat, D. (2017). "The complexity of co-opetitive networks". Business Proc. Manag. Journal, 23(1), 176-195.

Vahlne, J. E. y Johanson, J. (2017). "From internationalization to evolution: The Uppsala model at 40 years”. Journal of International Business Studies, 48(9), 1087-1102. 